\title{
Complications of a Multiple Myeloma: Myeloma Kidney, Myeloma Lung, Plasmacytoma and a Pathological Fracture in a Patient with Acute Renal Failure Secondary to Multiple Myeloma of Complex Karyotype
}

Igor Romaniouk Jakovler ${ }^{1 *}$, Natalia Allende ${ }^{2}$, Corral Antonio ${ }^{3}$, Elizaveta Zorina ${ }^{4}$, lyad Abuward ${ }^{1}$, Nisrine Arhda ${ }^{1}$, Marta Pais Seijas $^{1}$, Anabertha Narvaez $^{1}$ and Candido Díaz Rodriguez ${ }^{1}$

${ }^{1}$ Department of Nephrology, University Hospital of Santiago, Spain

${ }^{2}$ Department of Nephrology, University Hospital of Son Espases, Spain

${ }^{3}$ Department of Nephrology, Miramar clinic, Spain

${ }^{4}$ Faculty of Medicine, University of Moscow, Russia

*Corresponding author: Igor Romaniouk Jakovler, Department of Nephrology, University Hospital of Santiago, Spain, Tel: 0034639021641; E-mail: igor9843@gmail.com

Received Date: August 10, 2018; Accepted Date: August 28, 2018; Published Date: September 5, 2018

Copyright: @ 2018 Jakovler IR, et al. This is an open-access article distributed under the terms of the Creative Commons Attribution License, which permits unrestricted use, distribution, and reproduction in any medium, provided the original author and source are credited.

\begin{abstract}
Multiple Myeloma is the second hematological neoplasm, with an incidence rate that varies from the series studied, but generally goes from 6.2 per 100000 individuals. Usually affects the elderly and typically diagnosed between the ages of $65-74$ years, with an estimated 5-year survival rate ranging from $34.5 \%$ to $49.6 \%$ from 2000 to 2008. Renal failure in multiple myeloma occurs frequently and it is present in $20-40 \%$ of cases at the time of diagnosis. The complications related to the myeloma are several and include: anemia, hypercalcemia, hyperuricemia, but also complete entities such as myeloma kidney, plasmacytoma and myeloma lung, related with the infiltration of free light chains in different organs and its rarely to see all those complications in one patient. Neurological involvement is usually the result of a radiculopathy or pathological fractures. Lung involvement is rare and occurs in around $5 \%$ of cases. We present a 70 -year old patient with several cardiovascular risk factors that suffers an acute renal failure with acute pulmonary edema due to Multiple Myeloma of complex karyotype complicated with, myeloma lung, plasmacytoma and a pathological fracture.
\end{abstract}

Keywords: Multiple myeloma; Complex karyotype; Plasmacytoma; Myeloma lung

\section{Introduction}

Multiple myeloma $(\mathrm{MM})$ is a plasma cell malignancy that characterized by the accumulation of malignant plasma cells within the bone marrow (BM). The direct interactions of MM cells with accessory cells and secreted cytokines activate signaling pathways mediating growth, survival, drug resistance and the migration of multiple myeloma cells as well as osteoclastogenesis and angiogenesis [1].

These cells secrete immunoglobulin in excess, usually: IgG 55\%, IgA $23 \%$, IgD $1 \%$, IgM, IgE, only rarely in $18 \%$ of chain cases light alone. The proliferation of Multiple Myeloma results in anemia. Other characteristic is that the MM cells is a secretion stimulating substances of osteoclasts and inhibitors of the osteoblasts leading to a destruction of the bone tissue. In some cases the expansion of this neoplasm can compress the spinal cord and produce neurological effects. A high rate of infections is present in this population as well and many times it is the first manifestation of the disease [2].

Renal disease in myeloma often presents as renal insufficiency and proteinuria. The spectrum of renal lesions that is seen in patients with myeloma include "myeloma kidney," or cast nephropathy; light chain (AL) amyloidosis; monoclonal Ig deposition disease; and, less frequently, cryoglobulinemic glomerulonephritis and proliferative glomerulonephritis. The filtered light chains may cause intrarenal obstruction in the distal nephron by co-aggregating with the carbohydrate moiety of Tamm-Horsfall glycoprotein, which is produced in the thick ascending limb of the loop of Henle. Tubular injury also occurs as a result of light-chain dose-dependent toxicity to the proximal tubule epithelial cells, resulting in tubular necrosis, and the casts and the tubular epithelial cells may contain rhomboid or needle-shaped crystals in cases that are associated with the Fanconi syndrome. Lung affection is rare and occurs in around $5 \%$ of cases, it is related to the infiltration of light chains at the level of the parenchyma. It is discussed rather it is a separate entity receiving the name of "myeloma lung" or just an infiltration of the light chain into the organ [3-5].

The treatment usually is of the underlying disease.

\section{Case Report}

A 70-year-old patient who came to the emergency room in October 2017 due to a respiratory distress and fatigue of days of evolution. As background of interest the patients presented: grade I obesity, sleep apnea syndrome, community pneumonia in 2009, long-standing hypertension, cholecystectomy, type 2 diabetes mellitus with more than 10 years of evolution with negative eye fundus for diabetic retinopathy, severe aortic stenosis with valve replacement in 2015. The patient denies intake of toxins, Non-steroidal anti-inflammatory drugs (NSAIDs), diarrhea or vomiting the days before the admission. His last revision by a general practitioner 6 month prior to admission, reported a normal renal function. Physical examination revealed soft edemas to the root of the thighs and bilateral crepitation in both lung fields. Initial analysis: hemoglobin $6.6 \mathrm{mg} / \mathrm{dL}$, hematocrit $19 \%$, leukocytes $5400 \mathrm{~mm} 3$, platelets 73,000 , prothrombin activity $81 \%$, INR 1.1 , 
Citation: Jakovler IR, Allende N, Antonio C, Zorina E, Abuward I, et al. (2018) Complications of a Multiple Myeloma: Myeloma Kidney, Myeloma Lung, Plasmacytoma and a Pathological Fracture in a Patient with Acute Renal Failure Secondary to Multiple Myeloma of Complex Karyotype. J Nephrol Ther 8: 317. doi:10.4172/2161-0959.1000317

Page 2 of 4

activated partial thromboplastin time 166.1 seconds, calcium 8.3 $\mathrm{mg} / \mathrm{dL}$, potassium $6.3 \mathrm{mEq} / \mathrm{L}$, phosphorus $11 \mathrm{mg} / \mathrm{dL}$, urea $200 \mathrm{mg} / \mathrm{dL}$, creatinine $9 \mathrm{mg} / \mathrm{dL}$ and a glomerular filtration rate of $3 \mathrm{ml} / \mathrm{min} / \mathrm{m} 2$. The urinary analysis reported: an abundant proteinuria and glycosuria. The patient is admitted to the intensive care unit with a diagnosis of acute renal failure without clear cause and acute pulmonary edema starting immediately on hemodialysis through a femoral temporary venous catheter, improving the patients general condition. The urinary output was of $570 \mathrm{ml} / 24$ hours which improved greatly after several sessions of hemodialysis. Subsequent studies revealed a monoclonal band, the immunofixation showed a kappa free light chains 1.03 $\mathrm{mg} / \mathrm{dL}$, lambda free light chains $16.8 \mathrm{mg} / \mathrm{dL}$. The autoimmunity was negative. A 24 hour urine analysis was positive for a proteinuria of $7 \mathrm{~g}$ in 24 hours and a ratio of albumin creatinine in urine was of $4.6 \mathrm{mg} / \mathrm{gr}$. A bone marrow biopsy was done with the diagnosis of multiple myeloma LC. Lambda, stage III-B, the patient was seen by hematology who request a genetic study (Figure 1). It was decided by hematologist to begin the treatment with bortezomib and dexamethasone. The patient continued on a high flux hemodialysis using "Toray Bk-2.1" filters, available in our unit, with a dialysis time of 6 hours with filter replacement at 3 hours of dialysis during the month of October and half of November 2017. There decrease in light chains was observed throughout those months and a gradual improvement in renal function from 9 to $3 \mathrm{mg} / \mathrm{dL}$ of creatinine after nearly two month of admission. During the admission a total of 23 packed red blood cells were transfused. At the end of November 2017, the result of the following analysis was made with the next results: hemoglobin of $10.9 \mathrm{mg} / \mathrm{dL}$, a GFR of $42 \mathrm{ml} / \mathrm{min} / \mathrm{m} 2$, creatinine of $1.6 \mathrm{mg} / \mathrm{dL}$, proteinuria of $2.9 \mathrm{~g}$ in 24 hours. The patient was stable, maintaining good renal function and a diuresis of $3000 \mathrm{~mL}$ in 24 hours.

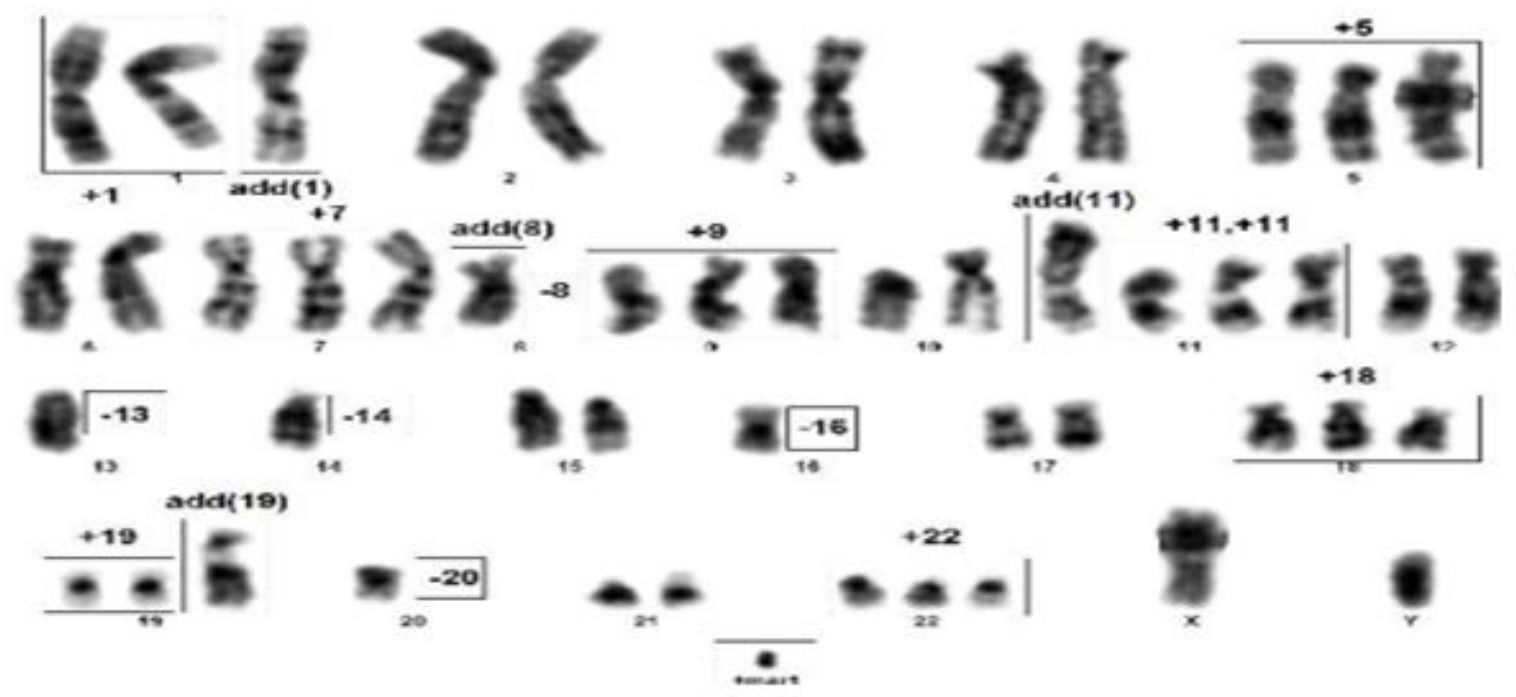

Figure 1: Bone marrow karyotype of a patient with multiple myeloma. A complex karyotype is observed (Chromosome formula: 51,XY, $+1, \operatorname{add}(1)(\mathrm{p} 13),+5,+7,-8, \operatorname{add}(8)(\mathrm{q} 22),+9,+11,+11, \quad \operatorname{add}(11)(\mathrm{p} 11.2),-13,-14,-16,+18,+19$, add $(19)(\mathrm{q} 13.3),-20, \quad+22,+$ mar1 $[10] / 52, \mathrm{idem},-$ $\operatorname{add}(1), \operatorname{dic}(1 ; 7)(\mathrm{p} 13 ; \mathrm{q} 36),+8,-\operatorname{add}(8)(\mathrm{q} 22), \operatorname{add}(8) \mathrm{p} 11.2),-11,-\operatorname{add}(11),+14,+14, \operatorname{add}(14)(\mathrm{q} 32),-\operatorname{mar} 1,+\operatorname{mar} 2[5] / 46, \mathrm{XY}[5])$.

In the beginning of December 2017, the patient presented a mild respiratory distress and cough without expectoration, analytically no data of active infection was observed nor condensation in chest X-ray seen. Upon the request and consultation of a pneumologyst, a computed tomography (Figure 2A) was done, with the radiological diagnosis of myeloma lung. Bronchoalveolar lavage requested does not confirms nor rejects the diagnosis. At the same time, the patient complains of a severe cervical pain that does not improve with usual analgesia, being diagnosed, as a pathology fracture of L1. The continue pain and the lack of response to the treatment, requires to perform a magnetic resonance detecting a plasmacytoma (Figure $2 \mathrm{~B}$ ), starting on radio therapy with slight improvement of the symptoms. Finally and after 6 month of treatment the patient was transferred to intensive care unit secondary to a respiratory sepsis due to probably hospital acquired pneumonia where after 24 hours he died. 
Citation: Jakovler IR, Allende N, Antonio C, Zorina E, Abuward I, et al. (2018) Complications of a Multiple Myeloma: Myeloma Kidney, Myeloma Lung, Plasmacytoma and a Pathological Fracture in a Patient with Acute Renal Failure Secondary to Multiple Myeloma of Complex Karyotype. J Nephrol Ther 8: 317. doi:10.4172/2161-0959.1000317

Page 3 of 4
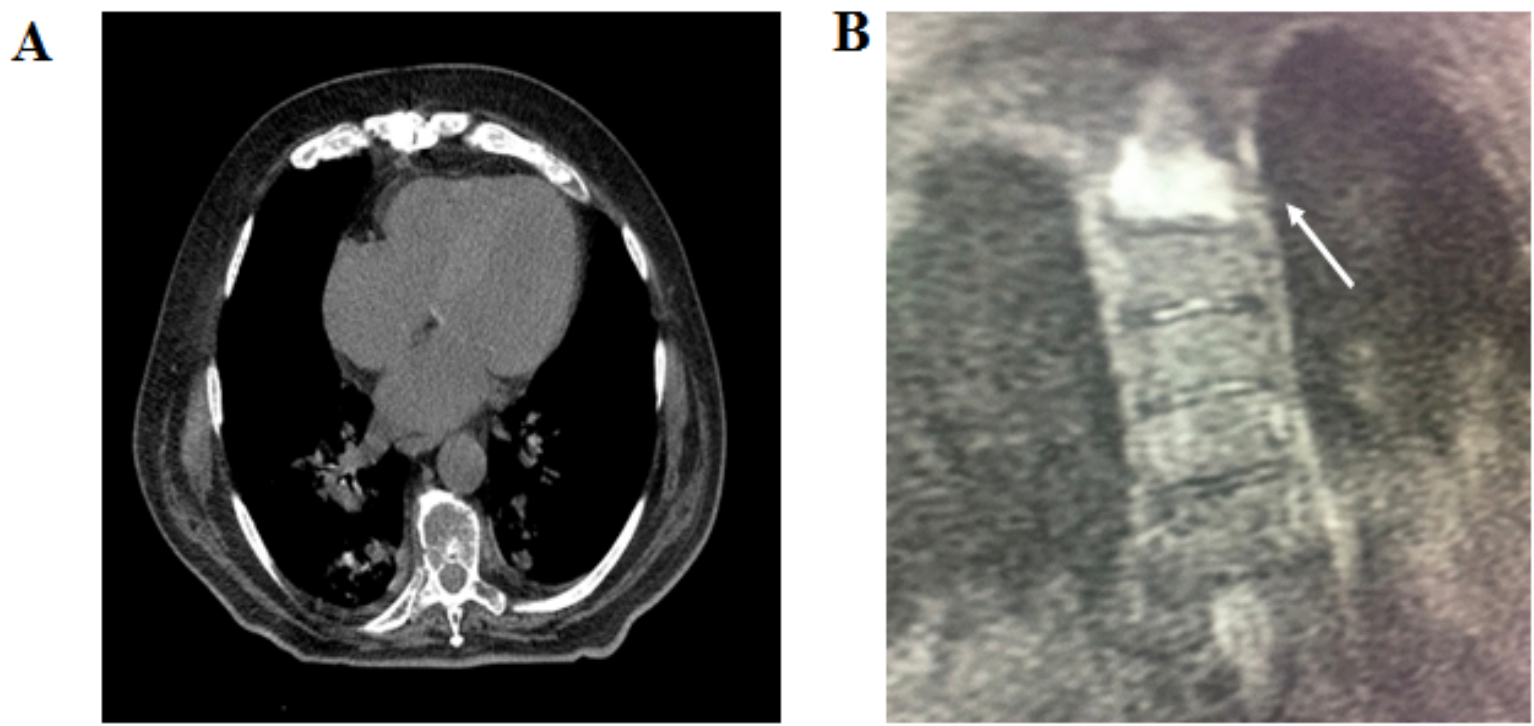

Figure 2: Tomography (A) and magnetic resonance (B) of a patient affected by multiple myeloma of complex karyotype. (A) Pulmonary involvement with basal interstitial infiltrates with micro-nodular pattern, in relation to the underlying disease, (B) probable plasmacytoma in the vertebral body of T6 with pathological fracture. Acute L1 fracture with probable intrinsic myeloma lesion.

\section{Discussion}

Multiple myeloma (MM) is a plasma cell malignancy characterized by the accumulation of malignant plasma cells within the bone marrow with an overall survival of the patient ranging from less than 1 year to over 20 years. Reliable risk stratification is a key to predicting outcome and guiding therapy. In 2015, the International Myeloma Working Group proposed the Revised International Staging System (R-ISS) for myeloma patients. Cytogenetic abnormalities in multiple myeloma affect every aspect of the disease, from evolution of the malignancy to clinical presentation, response to therapy and prognosis and a given abnormality may have a totally different meaning based on the disease stage. These abnormalities essentially classify multiple myeloma into several subtypes, moreover, they represent a unique cytogenetically distinct disease [6].

There are two main types of primary cytogenetic abnormalities in multiple myeloma: trisomies and translocations involving the immunoglobulin heavy chain ( $\mathrm{IgH})$ gene. The detection and interpretation of cytogenetic abnormalities in $\mathrm{MM}$ is of critical importance for prognosis and risk stratification of the disease. In one study, patients with $\mathrm{MM}$ without $\mathrm{t}(4 ; 14)$, $\operatorname{del}(17 \mathrm{p})$ or gain(1q21) who had stage I or II MM by the International Staging System (representing approximately $20 \%$ of all patients with MM) had an 8 -year survival of $75 \%$. In contrast, studies show that patients with high-risk cytogenetics have a median survival of $<2-3$ years despite best available treatments and are candidates for innovative, more aggressive clinical trials [7].

The patients presenting a complex karyotype are difficult to treat. Presenting both good and bad prognosis features. In the past this sort of patients was treated with plasmapheresis along with chemotherapy, the result was good always in the amount of light chains dropped to $50 \%$ with the treatment. The key to treating cast nephropathy is rapid lowering of free light chains, because this correlates with renal recover, never the chemotherapy alone.
Although the idea of eliminating free light chains (FLC) with plasmapheresis is attractive, the body distribution of the FLC, balanced between the intravascular and extravascular compartments and with $80 \%$ in the latter compartment, makes its elimination scarce with the usual plasma volume replacements (1, 5 times in about 2-3 hours). Given the very high cut-off that plasma filters have, higher rates of plasma exchange have the disadvantage that they are associated with the loss of other proteins of higher molecular weight that are essential for the organism and, therefore, it is not advisable to use these so aggressive therapies $[8,9]$.

Recently, several studies have been published on the efficiency in the elimination of FLC using a very high permeability, high-cut-off (HCO), designed for that purpose. The HCO membranes have a large pore, with a cut-off of $45-60 \mathrm{kD}$, which allows the filtration of both kappa and lambda light chains. The combination of filters with a high cut off with chemotherapy regimens based on bortezomib, reported recovery rates of renal function of $60-74 \%[8,9]$. However, there is an inconvenient of a very high cost of each filter that not all dialysis units can effort. The alternative is the extra-renal purification, which is a technique that combines convection, adsorption and diffusion. It uses a double chamber dialyzer: the first with a superflux polyphenylene membrane, which has a cut-off of $42 \mathrm{kD}$ and in which an ultrafiltration is carried out, and the second with the same membrane, but with low permeability, where it is made diffusion. The ultra-filtrate obtained in the first chamber passes through a resin cartridge where adsorption occurs and is reinfused before the second chamber. This technique is used in HD patients due to its high adsorption capacity of toxins bound to proteins, with the great advantage that it does not adsorb albumin. Since the cut-off of $42 \mathrm{kD}$ theoretically allows the passage of free light chains. It is important to highlight that even if there are several studies that suggest that a high cut-off hemodialysis together with chemotherapy offer better results in comparison with 
Citation: Jakovler IR, Allende N, Antonio C, Zorina E, Abuward I, et al. (2018) Complications of a Multiple Myeloma: Myeloma Kidney, Myeloma Lung, Plasmacytoma and a Pathological Fracture in a Patient with Acute Renal Failure Secondary to Multiple Myeloma of Complex Karyotype. J Nephrol Ther 8: 317. doi:10.4172/2161-0959.1000317

Page 4 of 4

conventional techniques, there are no randomized large studies to confirm so [10].

Our patient, with acute renal failure and pulmonary edema underwent the dialysis treatment with a high flux filter "Toray Bk-2.1" filters, available in our unit, with a dialysis time of 6 hours with filter replacement at 3 hours of dialysis. The filter was chosen mainly to its cost, studies, the theoretical reduction of free light chains and the experience in other centers (reference hospital) using the same filter in acute renal failure due to multiple myeloma. The analysis of free light chains was done prior and after the treatment, reporting a decrease of light chains in at last 30-40\% after each session together with chemotherapy with Bortezomib and dexamethasone. However, after 3 month several complications started, including plasmocytoma treated with radiotherapy, a pathological fracture and a highly discussed entity called "the myeloma lung" considered by many authors as only a mere complication of the light chain myeloma affecting the lungs and not a separate entity. In spite of some characteristics of the myeloma karyotype showed good prognosis, certain deletions implied a rapid progression of the disease. Despite a remarkable initial improvement, the patient presented a large number of complications, dying finally within a period of 6 months after the diagnosis and the treatment. These suggest that a complex karyotype is probably subsidiary to a more aggressive treatment, even having some favorable prognosis factors 10 .

\section{Conclusions}

The complications related to multiple myeloma are several, related mainly to the infiltration of light chains into different organs. The list includes anemia, hypercalcemia, hyperuricemia, but also complete entities such as myeloma kidney, plasmacytoma. Neurological involvement is usually the result of a radiculopathy or pathological fractures. Lung involvement is rare and occurs in around 5\% of cases, it is related to the infiltration of light chains at the level of the parenchyma. A complex karyotype, even having some favorable prognosis features, probably is subsidiary to a more aggressive treatment. The treatment of all these complications is of the underlying disease, the multiple myeloma.

\section{References}

1. Riveiro V, Ferreiro L, Toubes ME, Lama A, Álvarez-Dobaño JM, et al. (2018) Characteristics of patients with myelomatous pleural effusion. A systematic review. Rev Clin Esp 218: 89-97.

2. Chen H, Li P, Xie Y, Jin M (2018) Cytology and clinical features of myelomatous pleural effusion: Three case reports and a review of the literature. Diagn Cytopathol 46: 604-609.

3. Goranova-Marinova V, Yaneva M, Deneva T, Goranov S, Mužina Mišić D (2017) Multiple myeloma with advanced bone disease and low tumor burden-Different clinical presentation but similar outcome after Bortezomib-Based therapy and radiotherapy. Acta Clin Croat 56: 262-269.

4. Li L, Tong M, Zhao YT, He Y, Zhou HY, et al. (2018) Membrane translocation of Bruton kinase in multiple myeloma cells is associated with osteoclastogenic phenotype in bone metastatic lesions. Medicine (Baltimore) 97: e9482.

5. Iida S, Wakabayashi M, Tsukasaki K, Miyamoto K, Maruyama D, et al. (2018) Bortezomib plus dexamethasone versus thalidomide plus dexamethasone for relapsed or refractory multiple myeloma. Cancer Sci 109: 1552-1561.

6. Zanabili Al-Sibai J, Sánchez-García S, Rubio-Solís D (2017) Multiple myeloma: Presenting as intracranial plasmacytoma. Rev Clin Esp 217: 170-170.

7. Vázquez FJ, Funtowicz G, Rivello HG, Schutz N, Fantl D, et al. (2010) Hepatic nodular involvement secondary to multiple myeloma. Medicine 70: 311-315.

8. Romero-Espinoza, Pavel Barrera-Chairez, Esperanza Barros-Núñez, Patricio (2015) DNA methylation in multiple myeloma. Mexican Oncology Gazette 14: 229-230.

9. Braggio E, Garramuño FA (2013) The use of genetic alterations in the risk stratification of multiple myeloma. Medicine 73: 369-375.

10. Finkel KW, Cohen EP, Shirali A, Abudayyeh A, American Society of Nephrology Onco-Nephrology Forum (2016) Paraprotein-related kidney disease: Evaluation and treatment of Myeloma Cast Nephropathy. Clin J Am Soc Nephrol 11: 2273-2279. Epub Aug 15. 